\title{
Developing Hyperpolarized C-13 Spectroscopy and Imaging for Metabolic Studies in the Isolated Perfused Rat Heart
}

\section{Journal Article}

Author(s):

Weiss, Kilian; Mariotti, Erika; Hill, Deborah K.; Orton, Matthew R.; Dunn, Joel T.; Medina, Rodolfo A.; Southworth, Richard; Kozerke, Sebastian; Eykyn, Thomas R.

Publication date:

2012-07

Permanent link:

https://doi.org/10.3929/ethz-b-000052145

Rights / license:

In Copyright - Non-Commercial Use Permitted

Originally published in:

Applied Magnetic Resonance 43(1-2), https://doi.org/10.1007/s00723-012-0349-2 


\title{
Developing Hyperpolarized ${ }^{13} \mathrm{C}$ Spectroscopy and Imaging for Metabolic Studies in the Isolated Perfused Rat Heart
}

\author{
Kilian Weiss • Erika Mariotti • Deborah K. Hill • Matthew R. Orton • \\ Joel T. Dunn • Rodolfo A. Medina $\cdot$ Richard Southworth • \\ Sebastian Kozerke • Thomas R. Eykyn
}

Received: 1 February 2012/Revised: 26 April 2012/Published online: 19 May 2012

(C) Springer-Verlag 2012

\begin{abstract}
Hyperpolarized ${ }^{13} \mathrm{C}$ magnetic resonance is a powerful tool for the study of cardiac metabolism. In this work, we have implemented protocols for the realtime hyperpolarized ${ }^{13} \mathrm{C}$ investigation of Langendorff-perfused rat hearts using both non-selective non-localized spectroscopy and fast spectroscopic imaging. Following $\left[1-{ }^{13} \mathrm{C}\right]$ pyruvate infusion, we observed both catabolic and anaplerotic metabolic processes resulting in a number of metabolites, including bicarbonate, carbon dioxide, lactate, alanine and aspartate. Employing fast spectroscopic imaging, we were able to observe regional variations in pyruvate perfusion as well as in lactate and bicarbonate production.
\end{abstract}

\section{Introduction}

The Langendorff-perfused heart is an ex vivo model that allows the investigation of cardiac function and metabolic processes in a strictly controlled environment [1].

K. Weiss and E. Mariotti contributed equally to this work.

\footnotetext{
E. Mariotti · J. T. Dunn · R. A. Medina · R. Southworth · S. Kozerke ( $\square)$.

T. R. Eykyn $(\bowtie)$

Division of Imaging Sciences and Biomedical Engineering, King's College London,

St Thomas Hospital, London, UK

e-mail: kozerke@biomed.ee.ethz.ch

T. R. Eykyn

e-mail: thomas.eykyn@kcl.ac.uk

K. Weiss - S. Kozerke

Institute for Biomedical Engineering, University and ETH Zurich,

Gloriastrasse 35, 8092 Zurich, Switzerland

D. K. Hill · M. R. Orton - T. R. Eykyn

CRUK and EPSRC Cancer Imaging Centre, Royal Marsden NHS Trust,

The Institute of Cancer Research, Sutton, UK
} 
Various physiological and pathophysiological conditions of the heart can be reproduced using this model by direct control of its environment, substrate supply or energy requirements. Moreover, it provides the possibility to investigate the effect of endogenous and exogenous substances on cardiac metabolism, making it a key pharmacological research tool. In combination with appropriate non invasive methods like magnetic resonance spectroscopy (MRS) and imaging, it is a powerful preclinical approach for basic research, allowing the assessment of the effects of various cardiac interventions, such as small molecule inhibitors [2], up- and downregulation of signal transduction pathways [3] and response to ischemia-reperfusion injury [4].

Magnetic resonance spectroscopy can give insight into the role of cardiac metabolism under various conditions. In the perfused heart ${ }^{31} \mathrm{P}$ MRS is traditionally employed to measure intracellular concentrations of adenosine triphosphate (ATP) and phosphocreatine (PCr) [5-7]. ${ }^{1} \mathrm{H}$ MRS has been previously used to measure total creatine content [8] and triglyceride accumulation in ischemic myocardium [9], whereas ${ }^{13} \mathrm{C}$ MRS can be used to measure glucose oxidation [10]. Both ${ }^{31} \mathrm{P}$ and ${ }^{1} \mathrm{H}$ MRS have been translated to clinical application, for the quantification of pathophysiological changes in myocardial high-energy phosphate metabolism, creatine and triglyceride concentrations in patients, which can be affected by aortic stenosis [11], cardiomyopathy [12], ischemia [13] or diabetes [14, 15]. However, due to its relatively low sensitivity, the range of metabolites which are quantifiable by MRS for dynamic in vivo investigation are limited.

The recently introduced method of dissolution dynamic nuclear polarization (DNP) provides a possibility to enhance the MRS signal from nuclei by the order of tens of thousands [16], to facilitate the measurement of the kinetics of metabolic processes in healthy or diseased tissue previously inaccessible to nuclear magnetic resonance (NMR). Hyperpolarized $\left[1-{ }^{13} \mathrm{C}\right]$ pyruvate infusion has previously been shown to allow the measurement of pyruvate dehydrogenase activity (PDH) ex vivo in the perfused rat heart $[17,18]$ and in vivo under conditions such as diabetes and hypertrophy $[19,20]$. Hyperpolarized $\left[2-{ }^{13} \mathrm{C}\right.$ ] pyruvate can be used to assess the Krebs cycle showing significant decreases of TCA cycle intermediates, such as ${ }^{13} \mathrm{C}$ citrate and ${ }^{13} \mathrm{C}$ glutamate, in response to ischemia [21]. Moreover, PDH and TCA cycle activity can be assessed simultaneously through the use of hyperpolarized $\left[1,2-{ }^{13} \mathrm{C}_{2}\right.$ ] pyruvate [22]. Fatty acid metabolism has also been probed through the buffering of hyperpolarized acetate with acetyl carnitine in response to recovery from periods of ischemia [23] or through the addition of water soluble fatty acids such as octanoate [17].

While information from non-selective MRS is sufficient for estimating metabolic changes and pathologies with global effects, spatially resolved spectroscopy is needed to study local effects in the heart. Magnetic resonance spectroscopic imaging (MRSI) potentially provides the capacity to study local effects in the heart, allowing the characterization of heterogeneous metabolic changes and pathologies such as found in ischemic heart disease [24-26].

MRSI of hyperpolarized material poses additional challenges compared with that of more traditional thermally polarized ${ }^{1} \mathrm{H}$ MRSI. The rapidly decaying nature of hyperpolarization to thermal equilibrium and the metabolic conversion by enzymatic reactions require fast spectroscopic imaging techniques. The hyperpolarized 
magnetization needs to be traded between individual images and the number of dynamics in the imaging series. To preserve the magnetization for all dynamics, a low number of signal excitations in combination with small flip angles are desired. At the same time sufficient signal-to-noise ratio (SNR) needs to be assured. Various approaches have been proposed to address these challenges [27, 28] and in vivo applications of these techniques have been demonstrated in pig hearts [29].

In the present work an experimental set up for hyperpolarized ${ }^{13} \mathrm{C}-\mathrm{MR}$ spectroscopy and imaging was implemented in perfused rat hearts. In our experiments we detected the metabolic conversion from pyruvate to lactate, alanine, $\mathrm{HCO}_{3}{ }^{-}$and $\mathrm{CO}_{2}$, as well as a previously unobserved conversion from pyruvate to aspartate attributed to anaplerotic metabolism via the action of pyruvate carboxylase. To address the need for spatial resolution, fast spectroscopic imaging was implemented using echo planar spectroscopic imaging (EPSI) [30] allowing dynamic visualization of the spatial distribution of bicarbonate and lactate metabolites. Regional heterogeneity of these metabolite signals was observed.

\section{Experimental Methods}

\subsection{Isolated Heart Perfusion}

All animal experiments were carried out in accordance with local regulations [Home Office Guidance on the Operation of Animals (Scientific Procedures) Act 1986, HMSO (London)]. Male Wistar rats (250-300 g) underwent terminal anesthesia by intraperitoneal injection of $0.4 \mathrm{~mL}$ of sodium pentobarbitone. After assuring the absence of pedal pain withdrawal reflexes, $0.2 \mathrm{~mL}$ of heparin was injected into the femoral vein. The heart was harvested and placed in cold Krebs-Henseleit buffer to temporarily arrest it and minimize ischemic injury [31]. The isolated heart was cannulated to re-establish vascular perfusion [32] employing a plastic cannula $(\varnothing=3 \mathrm{~mm})$ inserted into the aorta and perfused with modified Krebs-Henseleit buffer $\left(\mathrm{NaCl} 118 \mathrm{mM}, \mathrm{NaHCO}_{3} 25 \mathrm{mM}, \mathrm{MgSO}_{4} 1.2 \mathrm{mM}, \mathrm{KCl} 5.9 \mathrm{mM}, \mathrm{Na}_{2}\right.$ EDTA $0.6 \mathrm{mM}$, glucose $11.1 \mathrm{mM}, \mathrm{CaCl}_{2} 2.5 \mathrm{mM}, \mathrm{pH} \sim 7.4$, continuously oxygenated by bubbling $95 \% \mathrm{O}_{2}, 5 \% \mathrm{CO}_{2}$ ) at a constant flow of $14 \mathrm{ml} / \mathrm{min}$ to achieve a constant pressure at the coronary arteries of $76 \mathrm{mmHg}$ (monitored by an in-line pressure transducer). Temperature was maintained at $37{ }^{\circ} \mathrm{C}$ using a thermostatically controlled water-jacketed system.

\subsection{DNP}

$14 \mu \mathrm{l}$ of $\left[1-{ }^{13} \mathrm{C}\right]$ pyruvic acid containing $15 \mathrm{mM}$ trityl radical and $1 \mathrm{mM}$ Gadolinium (Gd) (Dotarem) was hyperpolarized in a HyperSense ${ }^{\circledR}$ (Oxford Instruments) DNP at $3.35 \mathrm{~T}$ and $1.4 \mathrm{~K}$ for $\sim 1 \mathrm{~h}$. Dissolution was performed employing $4 \mathrm{ml}$ of $40 \mathrm{mM}$ TRIS buffer. Pyruvic acid was neutralized by addition of a number of moles of $\mathrm{NaOH}$ equal to that of the pyruvate sample to the collection vessel. The solution state hyperpolarization was measured in a separate experiment to be of the order $P \approx 22 \%$ (extrapolated back to the instant of the dissolution), 
corresponding to an enhancement factor of $\approx 27,000$ measured with respect to the thermal signal of the same sample acquired under Ernst angle conditions after signal decay. Immediately following dissolution, $3 \mathrm{ml}$ of hyperpolarized $\left[1-{ }^{13} \mathrm{C}\right]$ pyruvate $(50 \mathrm{mM})$ was injected into the perfusion line at constant flow $(1 \mathrm{ml} / \mathrm{min})$ from an automated injector, resulting in a final concentration of hyperpolarized solution of $\sim 3.3 \mathrm{mM}$ pyruvate in the perfusion buffer at the arrival to the heart.

\subsection{MR Spectroscopy and Imaging}

All experiments were carried out on a vertical wide bore Bruker 9.4T Avance III spectrometer with the bore maintained at $37{ }^{\circ} \mathrm{C}$. The perfused heart was inserted in a glass tube ( $\varnothing=15 \mathrm{~mm}$, with a silicone bung) filled with Krebs-Henseleit buffer. Coronary perfusate was aspirated from the top by a gravity-fed siphon.

For non-selective spectroscopic measurements $(n=5)$, the tube containing the perfused heart was placed within the isocenter of a custom-built ${ }^{23} \mathrm{Na} /{ }^{13} \mathrm{C}$ saddle coil $(\varnothing=15 \mathrm{~mm})$ and inserted into the spectrometer. Shimming was carried out using the ${ }^{23} \mathrm{Na}$ signal (FWHM $13 \mathrm{~Hz}$ ) and then re-tuned to ${ }^{13} \mathrm{C}$. Acquisition of the NMR data commenced at the end of the dissolution and a time-series of ${ }^{13} \mathrm{C}-\mathrm{MR}$ spectra $(n=128)$ was acquired using a single acquisition per spectrum, a small flip angle pulse $\left(\theta=10^{\circ}\right)$ and a time resolution of $2 \mathrm{~s} .{ }^{13} \mathrm{C}$-MRS data were processed and analyzed using TopSpin (Bruker). All spectra were baseline corrected and integrals of each peak extracted as a function of time. To estimate variability between measurements, we summed the entire time-series of spectra for each heart, estimating the total area under the metabolite peak, normalized to the total pyruvate area, as well as the ratio $\mathrm{HCO}_{3}{ }^{-} / \mathrm{CO}_{2}$ and the corresponding $\mathrm{pH}$. In the imaging experiments $(n=2)$ a dual-tuned ${ }^{1} \mathrm{H} /{ }^{13} \mathrm{C}$ birdcage coil with $20 \mathrm{~mm}$ inner diameter was employed. Shimming was performed on the water signal using the ${ }^{1} \mathrm{H}$ channel of the coil (FWHM $35 \mathrm{~Hz}$ ). Standard ${ }^{1} \mathrm{H}$ cine images were acquired in short axis (Fig. 1a) and four-chamber view (Fig. 1b) for planning purposes using a self-gated gradient echo sequence [33] with the following parameters: TE: $2.9 \mathrm{~ms}$, TR: $8 \mathrm{~ms}$, acquisition matrix: $128 \times 128$, field of view: $32 \mathrm{~mm} \times 32 \mathrm{~mm}$, spatial resolution: $250 \mu \mathrm{m} \times 250 \mu \mathrm{m}$, slice thickness: $5 \mathrm{~mm}$, flip angle: $20^{\circ}, 10$ cardiac frames.
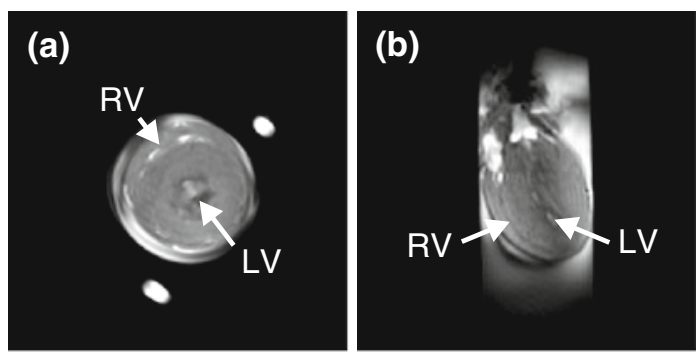

Fig. 1 a Time frame from a short axis ${ }^{1} \mathrm{H}$ cine image acquired as reference for the ${ }^{13} \mathrm{C}$ images. The left ventricle can be seen in the middle of the image. The right ventricle can be seen on the top left. The white dots outside the heart are incoming (bottom) and outgoing (top) perfusion line. b Four chamber view of the isolated perfused heart from a ${ }^{1} \mathrm{H}$ cine scan. The left ventricle can be seen on the right and the right ventricle on the left. The closed aortic valve is visible as a bright spot at the top of the left ventricle 


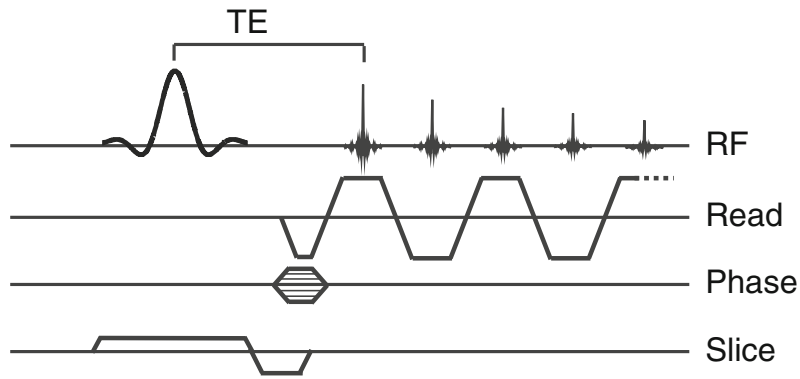

Fig. 2 Schematic diagram of the echo planar spectroscopic imaging (EPSI) sequence. Slice selective excitation is followed by phase encoding. The signal is acquired during an alternating gradient train

Hyperpolarized ${ }^{13} \mathrm{C}$ spectroscopic images were acquired using an echo planar spectroscopic imaging (EPSI) sequence as shown in Fig. 2. The acquisition parameters were as follows: TE: $1.315 \mathrm{~ms}$, TR: $89 \mathrm{~ms}$, time between dynamic images: $5 \mathrm{~s}$, acquisition matrix: $16 \times 16$, field of view: $32 \mathrm{~mm} \times 32 \mathrm{~mm}$, flip angle: $\approx 20^{\circ}$, spatial resolution: $2 \mathrm{~mm} \times 2 \mathrm{~mm}$, slice thickness: $5 \mathrm{~mm}$, spectral bandwidth during acquisition: 6,250 Hz, spectral resolution $12.2 \mathrm{~Hz}$. For each experiment 64 images were acquired in a total time of $320 \mathrm{~s}$ starting with the dissolution of the sample. MRSI data were reconstructed using Matlab (Mathworks Inc., Natick, MA). To avoid spectral N/2 ghosts, even and odd echoes of the echo planar readout were combined after linear phase correction of the even echoes, resulting in a spectral bandwidth of $3,125 \mathrm{~Hz}$. For spatial visualization of the distributions of pyruvate, lactate and bicarbonate, peak integrals were estimated on a pixel by pixel basis and overlaid as color-coded ${ }^{13} \mathrm{C}$ metabolite images to the ${ }^{1} \mathrm{H}$ reference images, interpolated to the same matrix size as the cine images. The color scales are normalized independently to the maximum intensity for each metabolite.

Spectra were averaged within regions of interest for a given time frame and phased using the pyruvate signal. The spectra reveal a linear phase due to the echo time of $1.315 \mathrm{~ms}$. This linear phase is taken into account by analyzing the data in the time domain using the AMARES function of the jMRUI software package [34, 35]. The resonances of pyruvate at $173 \mathrm{ppm}$, pyruvate hydrate at $181 \mathrm{ppm}$, lactate at $185 \mathrm{ppm}$ and bicarbonate at $162 \mathrm{ppm}$ in the individual time frames were fitted assuming Gaussian line profiles. For interpolation and display of the data signal time curves of pyruvate, lactate and bicarbonate estimated with AMARES were fitted using a bi-exponential function according to

$$
S(t)=a \times e^{-b \cdot t} \cdot\left(1-e^{-c \cdot t}\right),
$$

where $S$ is the observed signal and $a, b, c$ are free fitting parameters.

\section{Results}

A representative summed ${ }^{13} \mathrm{C}$ spectrum is shown in Fig. 3a, obtained by summing the entire hyperpolarized ${ }^{13} \mathrm{C}$ time-series acquired after injection of $\left[1-{ }^{13} \mathrm{C}\right]$ 
(a)

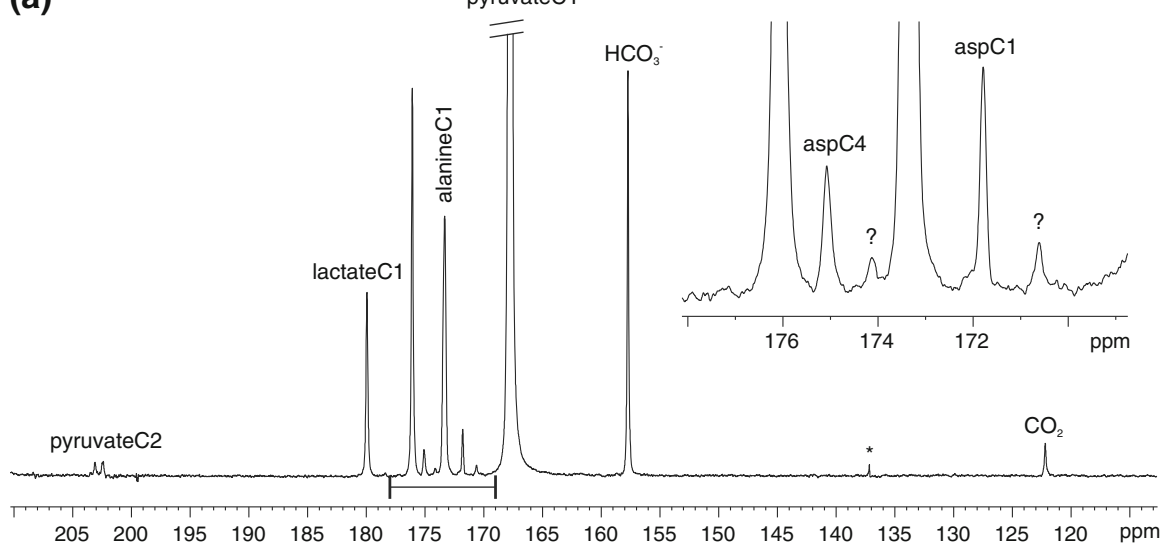

(b)
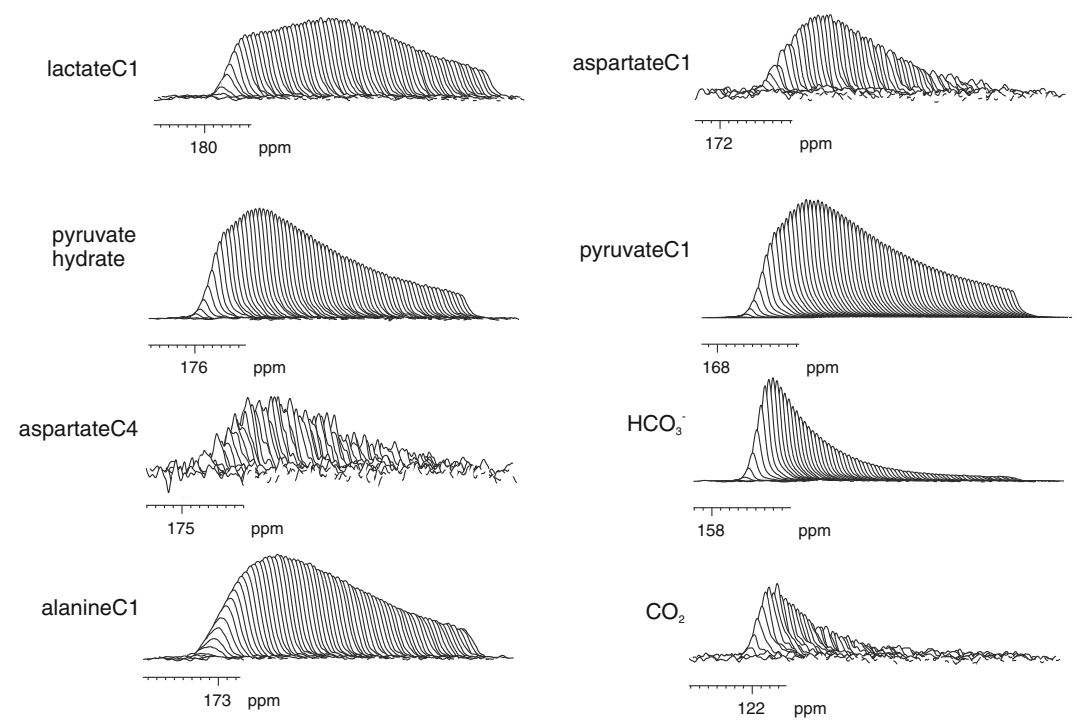

Fig. 3 a Example hyperpolarized spectrum obtained by summing the entire time-series (128 spectra) acquired as described in the "Experimental methods". Peaks correspond to pyruvate $\mathrm{C} 1$, natural abundance pyruvate $\mathrm{C} 2$ and pyruvate hydrate and the metabolites that are produced corresponding to lactate $\mathrm{C} 1$, alanine $\mathrm{C} 1, \mathrm{HCO}_{3}{ }^{-}, \mathrm{CO}_{2}$, aspartate $\mathrm{C} 1$ and aspartate $\mathrm{C} 4$ as well as some other minor metabolites not yet assigned as seen in the figure detail (top right). The peak $*$ is a noise spike from the coil. b Time series of ${ }^{13} \mathrm{C}-\mathrm{MR}$ spectra of hyperpolarized pyruvate $\mathrm{C} 1$ and its downstream metabolites shown in a

pyruvate. The spectrum shows a range of different metabolites that can be detected including signals arising from pyruvate $\mathrm{C} 1$, natural abundance pyruvate $\mathrm{C} 2$ and pyruvate hydrate and the metabolites that are produced by enzymatic conversion corresponding to lactate $\mathrm{C} 1$, alanine $\mathrm{C} 1, \mathrm{HCO}_{3}{ }^{-}, \mathrm{CO}_{2}$, aspartate $\mathrm{C} 1$ and aspartate $\mathrm{C} 4$ as well as some other minor metabolites not yet assigned as seen in the figure detail (top right). These minor peaks were not observed in the pyruvate preparation. 

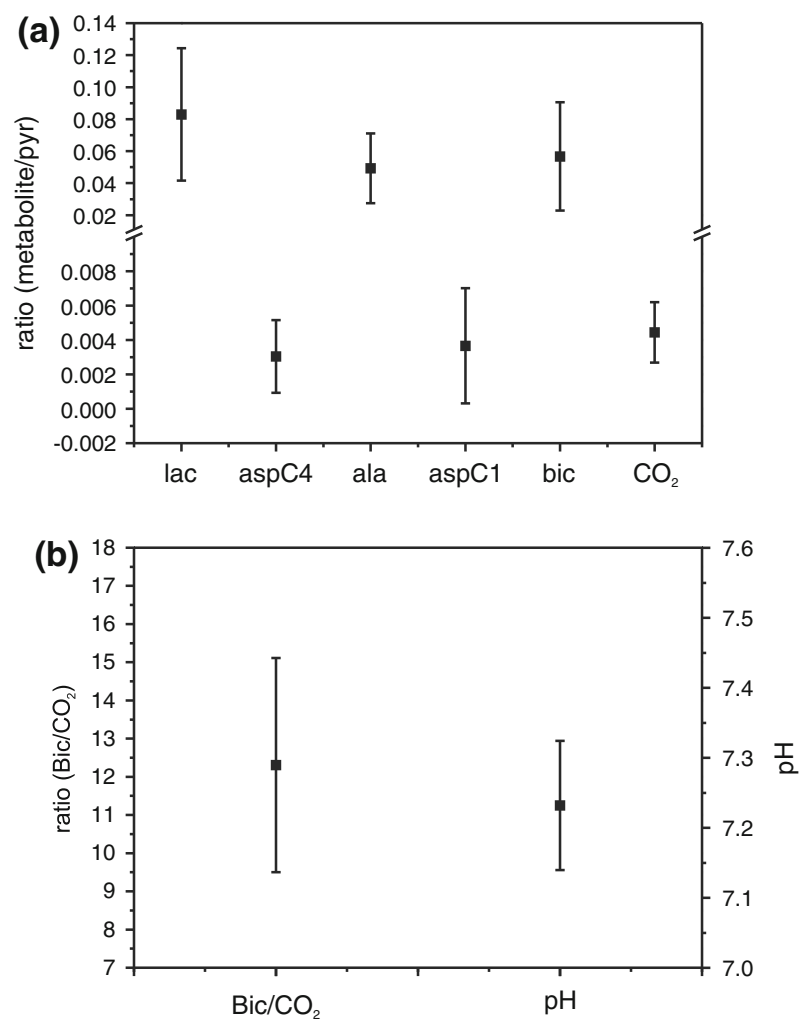

Fig. 4 a Mean and standard deviations $(n=5)$ of the total area under the curve formed by the peak integrals of lactate $\mathrm{C} 1$, aspartate $\mathrm{C} 4$, alanine $\mathrm{C} 1$, aspartate $\mathrm{C} 1, \mathrm{HCO}_{3}{ }^{-}$and $\mathrm{CO}_{2}$ normalized to the total pyruvate signal. b Means and standard deviations $(n=5)$ of the $\mathrm{HCO}_{3}{ }^{-} / \mathrm{CO}_{2}$ ratio and calculated $\mathrm{pH}$

Figure $3 \mathrm{~b}$ shows the temporal variation of each of these observed metabolite peaks displayed as a function of time.

Figure 4a presents the mean and standard deviation for the total area under each metabolite curve from the non-localized spectroscopic experiments $(n=5)$, corresponding to the peak integrals of lactate $\mathrm{C} 1$, aspartate $\mathrm{C} 4$, alanine $\mathrm{C} 1$, aspartate $\mathrm{C} 1, \mathrm{HCO}_{3}{ }^{-}$and $\mathrm{CO}_{2}$, normalized to the total pyruvate signal. Figure $4 \mathrm{~b}$ shows the mean and standard deviations of the $\mathrm{HCO}_{3}{ }^{-} / \mathrm{CO}_{2}$ ratio and corresponding $\mathrm{pH}$ calculated using the Henderson-Hasselbalch equation. The latter is consistent with the intracellular physiological $\mathrm{pH}$ in the heart measured previously [36].

Time series of images for pyruvate, lactate and bicarbonate from two individual experiments are shown in Fig. 5a and b overlaid onto a time frame of a ${ }^{1} \mathrm{H}$ cine image in short axis view (Fig. 1a). The temporal interval between the dynamic images is $5 \mathrm{~s}$. The time frame $t=0 \mathrm{~s}$ was chosen to be the last time frame without ${ }^{13} \mathrm{C}$ signal before the appearance of the hyperpolarized signal. Starting with injection of hyperpolarized solution into the perfusion line, the signal of pyruvate is visible in the inlet line indicated by the arrow in the pyruvate image in time frame $t=5 \mathrm{~s}$ (top of Fig. 5a). Signal in the outflow tube can be found in time frame 
(a)

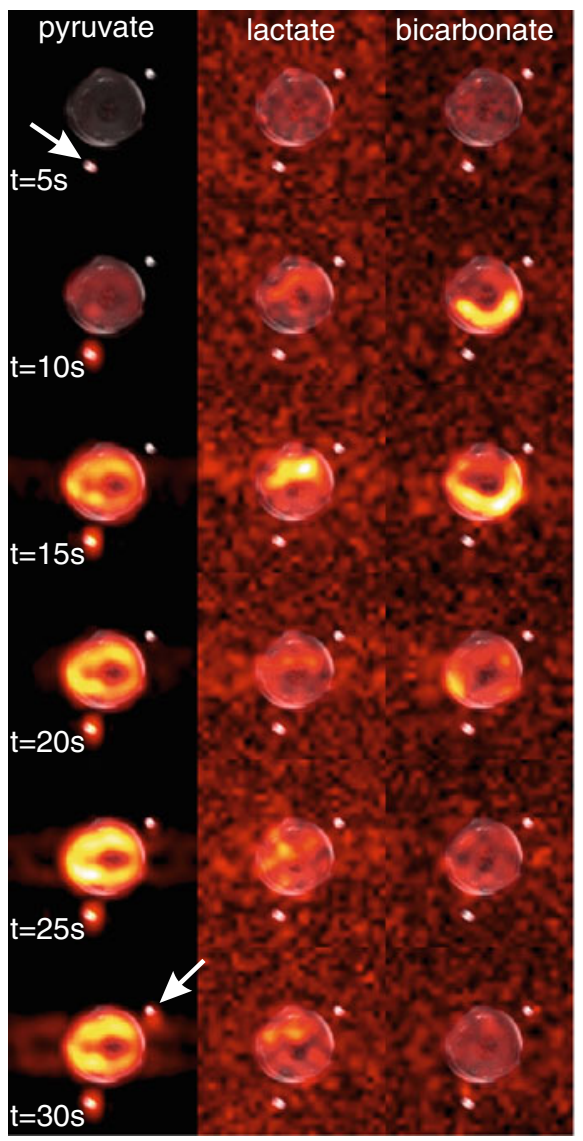

(c)

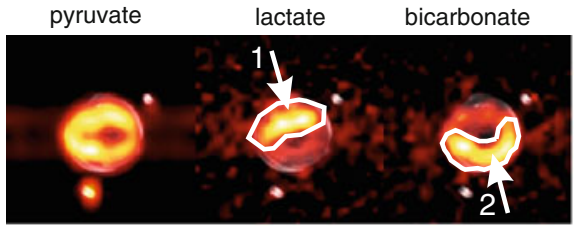

(b)

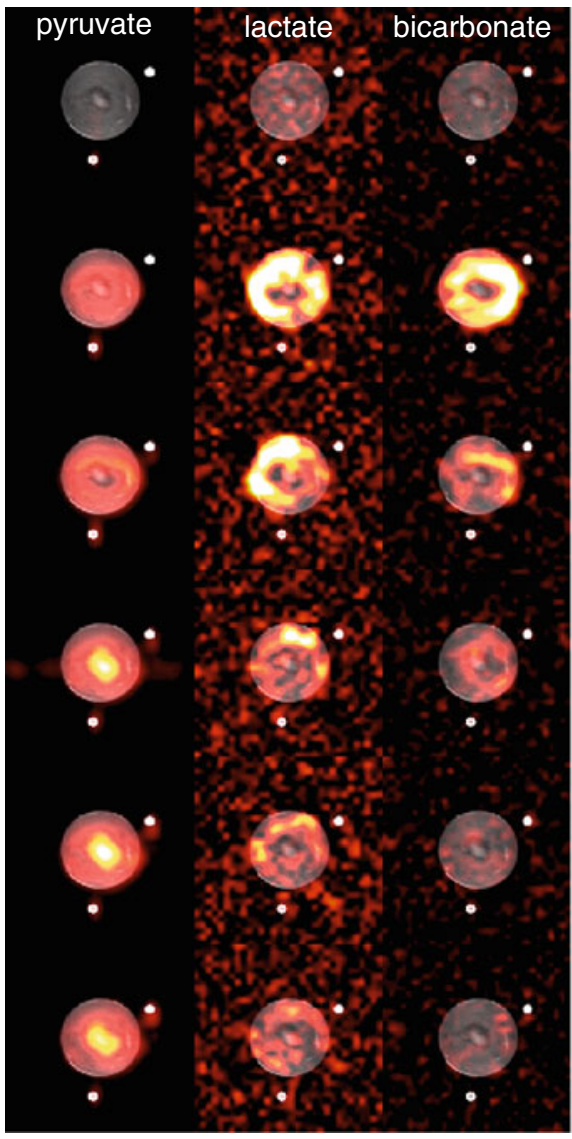

(d)

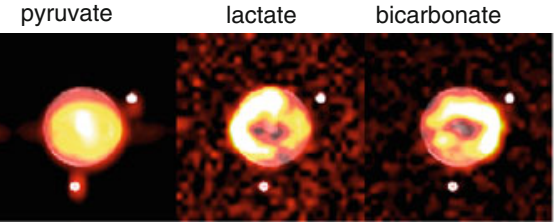

Fig. 5 a, b Time series of ${ }^{13} \mathrm{C}$ images from a slice in short axis view of the myocardium overlaid on the ${ }^{1} \mathrm{H}$ reference image (Fig. 1a). a and b show two different hearts. Images of pyruvate, lactate and bicarbonate signals are shown in the 1st, 2nd and 3rd columns, respectively. The temporal resolution between the images is $5 \mathrm{~s}$, where time $t=0 \mathrm{~s}$ is defined to be the last image without ${ }^{13} \mathrm{C}$ signal before appearance of hyperpolarized $\left[1-{ }^{13} \mathrm{C}\right]$ pyruvate. The arrow in time frame $t=5 \mathrm{~s}$ in a indicates the incoming perfusion line, the arrow in time frame $t=30 \mathrm{~s}$ in a indicates the outgoing tubing. c and d Mean images of time frames $t=10 \mathrm{~s}$ to $t=20 \mathrm{~s}$ from the image series in $\mathbf{a}$ and $\mathbf{b}$. The images show a clear heterogeneous distribution of lactate and bicarbonate signals in experiment 1 (a and c). In experiment 2 the distribution of lactate and bicarbonate is much more homogeneous $\mathbf{b}$ and $\mathbf{d}$. The region of high lactate and bicarbonate production in experiment 1 is indicated by arrow 1 and 2 respectively 
$t=30 \mathrm{~s}$, indicated by the arrow (bottom of Fig. 5a). The metabolic products lactate and bicarbonate are visible in the myocardium from time frame $t=10 \mathrm{~s}$. To further increase the SNR of the images the mean of the time points $t=5 \mathrm{~s}$ to $t=20 \mathrm{~s}$ is shown in Fig. 5c and d. It is apparent that in experiment 1 (Fig. 5a, c) the distributions of pyruvate perfusion and the production of lactate and bicarbonate are not homogeneous over the whole myocardium. In comparison, experiment 2 (Fig. 5b, d) shows a much more homogeneous distribution of the metabolic products lactate and bicarbonate. The regions indicated by the arrows 1 and 2 in the lactate and bicarbonate images of experiment 1 (Fig. 5c) reveal enhanced production of lactate and bicarbonate, respectively, representing different metabolic processes taking place in these regions. Time courses of pyruvate, lactate and bicarbonate signal amplitudes estimated with AMARES from the two regions of interest in experiment 1 and from the whole myocardium in experiment 2 are shown in Fig. 6a-c. Fitted spectra of the regions of high lactate production and high bicarbonate production of experiment 1 and the whole myocardium of experiment 2 are shown in Fig. 6d-f, together with fit residuals. Differences in the lactate resonance at $185 \mathrm{ppm}$ and the bicarbonate resonance at $162 \mathrm{ppm}$ between the two regions in experiment 1 and in experiment 2 are visible. Figure $6 g-i$ shows a stacked plot of the absolute spectra estimated with AMARES from the two regions of interest indicated in Fig. 5b. Overall the signals from spectroscopic imaging are clearly limited by SNR as can be seen in the images in Fig. 5 and the fitting residuals in Fig. 6d-f. Furthermore, the temporal resolution is lower compared with non-selective spectroscopy. However, the time courses of the signals are comparable between the two techniques.

\section{Discussion}

A schematic diagram of the metabolic pathways we observed is shown in Fig. 7. We observe conversion of hyperpolarized $\left[1-{ }^{13} \mathrm{C}\right]$ pyruvate to lactate catalyzed by lactate dehydrogenase (LDH), to alanine catalyzed by alanine transaminase (ALT), to bicarbonate catalyzed by $\mathrm{PDH}$ and interconversion of bicarbonate and $\mathrm{CO}_{2}$ catalyzed by carbonic anhydrase (CA). We also observe the conversion of hyperpolarized $\left[1-{ }^{13} \mathrm{C}\right]$ pyruvate to aspartate $\mathrm{C} 1$ and $\mathrm{C} 4$ by transamination via oxaloacetate. This is attributed to anaplerotic metabolism via the action of pyruvate carboxylase (PC). The $\mathrm{C} 1$ label appears from direct conversion of pyruvate via oxaloacetate. It has been suggested that the $\mathrm{C} 4$ signal in aspartate arises from TCA cycle conversion of oxaloacetate to malate and fumarate where the position of the hyperpolarized signal is scrambled due to molecular symmetry [37]. The aspartate C4 signal was less intense than the aspartate C1 signal. However, we did not observe oxaloacetate, malate or fumarate in any of our experiments, and therefore it was not possible to distinguish between this scrambling and possible direct carboxylation of pyruvate by hyperpolarized $\mathrm{CO}_{2}^{*}$. We further observed two unknown peaks that were not present as impurities in the pyruvate preparation. The one at $170.7 \mathrm{ppm}$ is consistent with asparagine whilst the identity of the peak at $174.2 \mathrm{ppm}$ remains to be determined. 


\section{Experiment 1}
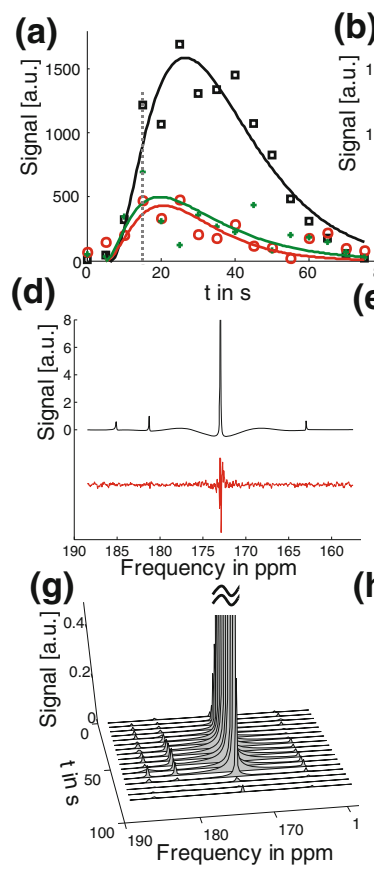

(b)

(h)

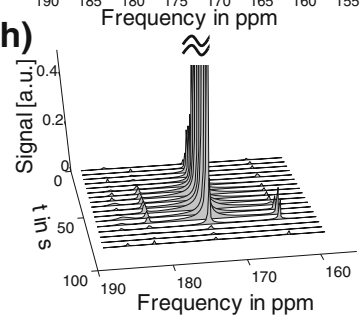

Experiment 2

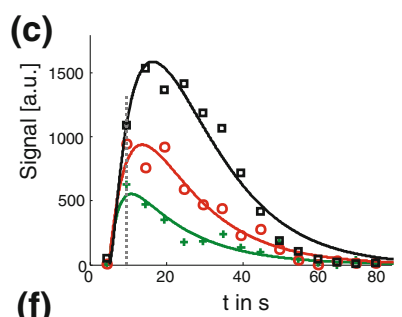

(f)

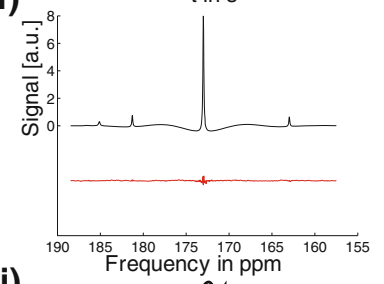

(i)

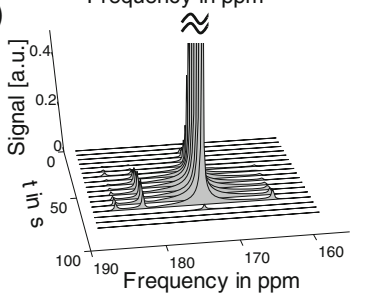

Fig. 6 Time courses of the pyruvate, lactate and bicarbonate signals, estimated with AMARES from the two experiments shown in Fig. 5. For experiment 1 data from the region of high lactate production (a) and the region of high bicarbonate production (b) indicated by arrow 1 and 2 in Fig. 5c was extracted. For experiment 2 mean data of the whole myocardium is shown. The solid lines show a fit of the data using a bi-exponential equation for interpolation and display purposes only. d-f AMARES fitted spectra and fit residual at the time point indicated by the dashed lines in a-c. d Average spectrum of nine voxels in the region of high lactate production of experiment 1. e Average spectrum of 15 voxels in the region of high bicarbonate production of experiment 1. f Average spectrum of all voxels in myocardium of experiment 2. Bottom shows stacked plots of the spectra of the time series estimated with AMARES from the two regions of interest in experiment $1(\mathbf{g}, \mathbf{h})$ and the whole myocardium in experiment 2 (i). For better visualization without baseline distortions due to the linear phase of the spectra, absolute values are shown

The aspartate $\mathrm{C} 1$ and $\mathrm{C} 4$ peaks which we observed have previously been described in liver metabolism, which typically exhibits a greater degree of anaplerosis than in the heart [37]. However, anaplerotic reactions are also essential to maintain TCA cycle intermediate levels constant in the heart, and we observe them here using DNP NMR for the first time. To date, anaplerosis has only been studied using ${ }^{13} \mathrm{C}$-isotopomer analysis by MR [38-40], but no real-time estimation of this important metabolic pathway has been carried out. Moreno et al. [38], used isotopomer analysis of heart homogenates (from hearts similarly perfused with 3 and $6 \mathrm{mM}$ pyruvate), demonstrating that $80 \%$ of acetyl CoA arose from pyruvate metabolism, whilst only $8-10 \%$ was attributed to anaplerosis. Using the relative amplitude of the aspartate $\mathrm{C} 1$ signal and the $\mathrm{HCO}_{3}{ }^{-}$signal, we estimate $\mathrm{PC}$ and $\mathrm{PDH}$ activity to be approximately $7 \%$ and therefore in good agreement. 


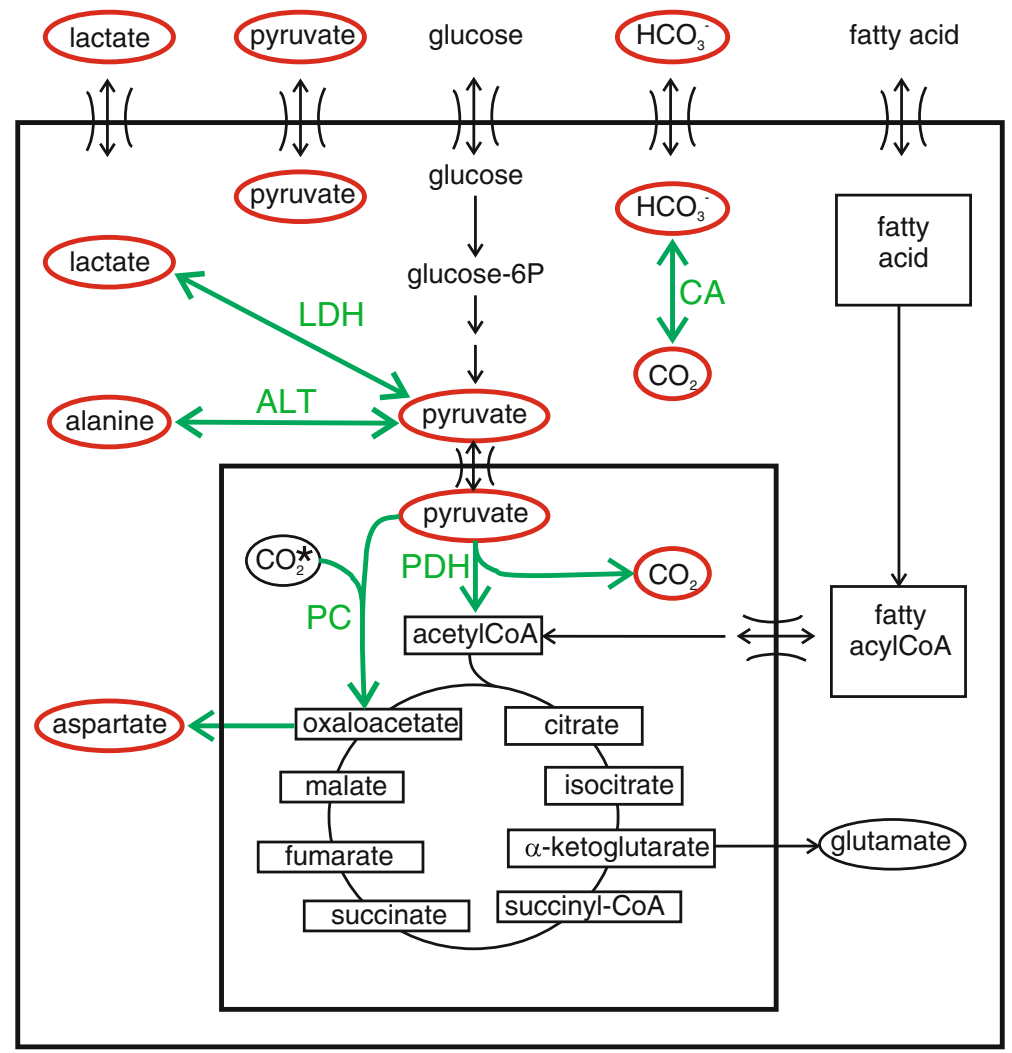

Fig. 7 Schematic representation of pyruvate metabolism with the metabolites observed using nonselective spectroscopy shown in red. We observe conversion of hyperpolarized $\left[1-{ }^{13} \mathrm{C}\right]$ pyruvate to lactate, alanine, bicarbonate, $\mathrm{CO}_{2}$ and aspartate under the action of the enzymes lactate dehydrogenase $(\mathrm{LDH})$, alanine transaminase (ALT), pyruvate dehydrogenase (PDH), pyruvate carboxylase (PC) and transamination of oxaloacetate to aspartate. The outer box corresponds to the plasma membrane whilst the inner box is the mitochondrial membrane. From our experiments we are not able to distinguish between pyruvate carboxylation via hyperpolarized $\mathrm{CO}_{2}^{*}$ or symmetrization of oxaloacetate via conversion to fumarate

Hyperpolarized tracers, such as $\left[1-{ }^{13} \mathrm{C}\right]$ pyruvate, may therefore be a useful tool for real-time investigation of anaplerotic metabolism in the perfused rat heart ex vivo with the potential to also be studied in vivo.

In the present work, fast spectroscopic imaging was implemented to dynamically image perfusion with pyruvate and to follow its conversion to lactate and bicarbonate in the isolated perfused heart. The early upslope of the bicarbonate signal in experiment 1 shown in Figs. 5a and 6b compared with the pyruvate signal is a visual effect due to the down scaling of the pyruvate signals. In fact, the upslope of the pyruvate signals is earlier than that of the bicarbonate signals. In experiment 1 the bicarbonate signal reaches its maximum point about $5 \mathrm{~s}$ earlier in region 2 (between $t=10 \mathrm{~s}$ and $t=15 \mathrm{~s}$ ) than with region 1 (between $t=15$ and $25 \mathrm{~s}$ ). The image series and the spectra of experiment 1 presented in Figs. 5 and 6 show a 
heterogeneous distribution of lactate and bicarbonate within the myocardium, indicated by the two regions in Fig. 5c. In contrast, the imaging series of experiment 2 display a more homogeneous distribution of metabolites. One may note also in this second experiment pyruvate signal arising within the left ventricle, suggesting partial opening of the aortic valve in this experiment. These regional differences that we observe are most likely due to regional variations in pyruvate perfusion and its subsequent metabolism. Thus, regional differences in the magnitude of pyruvate signal provide some insight into tissue perfusion, while regional differences in metabolic fate provide insight into the degree of metabolic derangement that a particular region may be experiencing due, for example, to ischaemia. Further investigation of these effects in well-controlled models of ischemia or metabolic perturbations is therefore needed. The heterogeneities in myocardial metabolism that we observe cannot be detected using non-selective spectroscopy (which averages these signals over the entire volume) and are a significant advantage of this approach.

There are currently some limitations in our methodology for both the nonselective spectroscopy and the spectroscopic imaging experiments. Even though all our experiments were performed following a standard protocol, some variability in the intensity of the metabolite peaks (Fig. 4a) was detected. Our experiments were carried out without monitoring cardiac function, meaning that we were unable to assess the consistency of our heart perfusion protocol after it is inserted into the spectrometer. A lack of ECG triggering leads to image blurring along the phase encoding direction (left, right), due to residual motion as seen in the ${ }^{13} \mathrm{C}$ images in Fig. 5a and b. In future experiments, the primary cardiac parameters (e.g., coronary flow, left ventricular developed pressure and heart rate) will be detected using a balloon inserted in the left ventricle connected to a pressure transducer. Moreover, heart rate (and thus energy demand) will be maintained constant using an MRcompatible pacing system, which is currently under construction. This system can also be employed to trigger image acquisition and minimize motion-related image blurring.

Cardiac metabolism is very sensitive to changes in substrate availability. The choice of the composition of the Krebs-Henseleit buffer is crucial to obtain physiologically relevant results [41]. In these experiments, the buffer contained glucose but not fatty acids, which has been previously shown to greatly affect the conversion of pyruvate, for example in the presence of octanoate [17]. However, physiological long-chain fatty acids bound to albumin confound hyperpolarized ${ }^{13} \mathrm{C}-\mathrm{MR}$ measurements due to a significant shortening of $\mathrm{T}_{1}$ [38]. Furthermore, in our experiments the concentration of pyruvate required to give sufficient signal-tonoise is more than ten times higher than physiological concentrations of pyruvate in blood [38], thus influencing substrate utilization in the heart. Further investigations are needed to understand the impact of this upon substrate selection in our isolated heart model.

Our imaging experiments are currently limited by sensitivity and therefore also by temporal resolution. Advanced methods to optimize excitation angles for pyruvate, lactate and bicarbonate independently using spatial spectral pulses in combination with EPSI [42] or single-shot EPI (echo planar imaging) [28] readout 
could be used in future work to overcome these limitations. Additionally, partial $\mathrm{k}$-space undersampling techniques exploiting spatiotemporal correlations such as k-t PCA [43] could be used to minimize the number of signal excitations and thus further accelerate image acquisition.

\section{Conclusion}

We have implemented protocols for real-time metabolic studies in the perfused rat heart employing both non-selective ${ }^{13} \mathrm{C}$ MR spectroscopy and slice-selective spectroscopic imaging for localized measurements. In agreement with previous studies [17, 18], we have shown that it is feasible to detect the conversion of hyperpolarized $\left[1-{ }^{13} \mathrm{C}\right.$ ] pyruvate to lactate, alanine, $\mathrm{HCO}_{3}{ }^{-}$and $\mathrm{CO}_{2}$ using non selective spectroscopy experiments. We have also demonstrated the conversion of hyperpolarized $\left[1-{ }^{13} \mathrm{C}\right]$ pyruvate to aspartate $\mathrm{C} 1$ and $\mathrm{C} 4$ which we attribute to anaplerotic metabolism. Information on the spatial distribution of hyperpolarized pyruvate, lactate and bicarbonate was obtained using slice-selective spectroscopic imaging employing an echo planar readout. Spatial distributions of the ${ }^{13} \mathrm{C}$ metabolite signals can be measured and heterogeneous metabolism within the myocardium can be detected. Fast spectroscopic imaging of hyperpolarized compounds is therefore a promising tool for the investigation of myocardial metabolism in the isolated perfused heart model and an exciting prospect for pharmacological studies.

Acknowledgments We are grateful to support provided by The Centre of Excellence in Medical Engineering funded by the Wellcome Trust and EPSRC under Grant number WT 088641/Z/09/Z, an Engineering and Physical Science Research Council studentship, the British Heart Foundation project Grant PG/10/020, a Royal Society research grant and the Swiss National Science Foundation, Grant \#CR3213_132671/1. We thank Craig Cummings for construction of the home-built ${ }^{13} \mathrm{C}$ coil and Dr. Steven Reynold for the loan of the dual tuned ${ }^{1} \mathrm{H} /{ }^{13} \mathrm{C}$ birdcage coil.

\section{References}

1. F.J. Sutherland, D.J. Hearse, Pharmacol. Res. 41, 613-627 (2000)

2. T.A. McKinsey, D.A. Kass, Nat. Rev. Drug Discover. 6, 617-635 (2007)

3. M. Mayr, U. Mayr, Y.L. Chung, X.K. Yin, J.R. Griffiths, O.B. Xul, Proteomics 4, 3751-3761 (2004)

4. D.J. Hausenloy, A. Tsang, D.M. Yellon, Trends Cardiovasc. Med. 15, 69-75 (2005)

5. J.M. Salhany, G.M. Pieper, S. Wu, G.L. Todd, F.C. Clayton, R.S. Eliot, J. Mol. Cell. Cardiol. 11, 601-610 (1979)

6. P.B. Garlick, G.K. Radda, P.J. Seeley, Biochem. Biophys. Res. Commun. 74, 1256-1262 (1977)

7. R. Southworth, P.B. Garlick, Am. J. Physiol. Heart Circ. Physiol. 285, H316-H324 (2003)

8. J.F. Unitt, J. Schrader, F. Brunotte, G.K. Radda, A.M. Seymour, Biochim. Biophys. Acta 1133, 115-120 (1992)

9. L.C. Stewart, J.K. Saunders, R. Deslauriers, D. Bourgeois, J.F.J. Nedelec, Magn. Reson. Med. 30, 655-660 (1993)

10. D.E. Hoekenga, J.R. Brainard, J.Y. Hutson, Circ. Res. 62, 1065-1074 (1988)

11. C.J. Hardy, R.G. Weiss, P.A. Bottomley, G. Gerstenblith, Am. Heart J. 122, 795-801 (1991)

12. M. Beer, T. Seyfarth, J. Sandstede, W. Landschutz, C. Lipke, H. Kostler, M. von Kienlin, K. Harre, D. Hahn, S. Neubauer, J. Am. Coll. Cardiol. 40, 1267-1274 (2002) 
13. P.A. Bottomley, R.G. Weiss, Lancet 351, 714-718 (1998)

14. M. Scheuermann-Freestone, P.L. Madsen, D. Manners, A.M. Blamire, R.E. Buckingham, P. Styles, G.K. Radda, S. Neubauer, K. Clarke, Circulation 107, 3040-3046 (2003)

15. J.M. McGavock, I. Lingvay, I. Zib, T. Tillery, N. Salas, R. Unger, B.D. Levine, P. Raskin, R.G. Victor, L.S. Szczepaniak, Circulation 116, 1170-1175 (2007)

16. J.H. Ardenkjaer-Larsen, B. Fridlund, A. Gram, G. Hansson, L. Hansson, M.H. Lerche, R. Servin, M. Thaning, K. Golman, Proc. Natl. Acad. Sci. USA. 100, 10158-10163 (2003)

17. M.E. Merritt, C. Harrison, C. Storey, F.M. Jeffrey, A.D. Sherry, C.R. Malloy, Proc. Natl. Acad. Sci. USA. 104, 19773-19777 (2007)

18. M.E. Merritt, C. Harrison, C. Storey, A.D. Sherry, C.R. Malloy, Magn. Reson. Med. 60, 1029-1036 (2008)

19. M.A. Schroeder, L.E. Cochlin, L.C. Heather, K. Clarke, G.K. Radda, D.J. Tyler, Proc. Natl. Acad. Sci. USA. 105, 12051-12056 (2008)

20. H.J. Atherton, M.S. Dodd, L.C. Heather, M.A. Schroeder, J.L. Griffin, G.K. Radda, K. Clarke, D.J. Tyler, Circulation 123, U2134-U2552 (2011)

21. M.A. Schroeder, H.J. Atherton, D.R. Ball, M.A. Cole, L.C. Heather, J.L. Griffin, K. Clarke, G.K. Radda, D.J. Tyler, FASEB J. 23, 2529-2538 (2009)

22. A.P. Chen, R.E. Hurd, M.A. Schroeder, A.Z. Lau, Y.-p. Gu, W.W. Lam, J. Barry, J. Tropp, C.H. Cunningham, NMR Biomed. 25, 305-311 (2012)

23. P.R. Jensen, T. Peitersen, M. Karlsson, R. in’t Zandt, A. Gisselsson, G. Hansson, S. Meier, M.H. Lerche, J. Biol. Chem. 284, 36077-36082 (2009)

24. K. Golman, J.S. Petersson, P. Magnusson, E. Johansson, P. Akeson, C.M. Chai, G. Hansson, S. Mansson, Magn. Reson. Med. 59, 1005-1013 (2008)

25. R. Southworth, J.L. Dearling, R.A. Medina, A.A. Flynn, R.B. Pedley, P.B. Garlick, Eur. J. Nucl. Med. Mol. Imaging 29, 1334-1341 (2002)

26. A.E. Arai, J. Cardiovasc. Pharmacol. Ther. 16, 313-320 (2011)

27. C.H. Cunningham, A.P. Chen, M.J. Albers, J. Kurhanewicz, R.E. Hurd, Y.F. Yen, J.M. Pauly, S.J. Nelson, D.B. Vigneron, J. Magn. Reson. 187, 357-362 (2007)

28. C.H. Cunningham, A.P. Chen, M. Lustig, B.A. Hargreaves, J. Lupo, D. Xu, J. Kurhanewicz, R.E. Hurd, J.M. Pauly, S.J. Nelson, D.B. Vigneron, J. Magn. Reson. 193, 139-146 (2008)

29. A.Z. Lau, A.P. Chen, N.R. Ghugre, V. Ramanan, W.W. Lam, K.A. Connelly, G.A. Wright, C.H. Cunningham, Magn. Reson. Med. 64, 1323-1331 (2010)

30. P. Mansfield, Magn. Reson. Med. 1, 370-386 (1984)

31. M. Skrzypiec-Spring, B. Grotthus, A. Szelag, R. Schulz, J. Pharmacol. Toxicol. Methods 55, 113-126 (2007)

32. R.M. Bell, M.M. Mocanu, D.M. Yellon, J. Mol. Cell. Cardiol. 50, 940-950 (2011)

33. E. Heijman, W. de Graaf, P. Niessen, A. Nauerth, G. van Eys, L. de Graaf, K. Nicolay, G.J. Strijkers, NMR Biomed. 20, 439-447 (2007)

34. L. Vanhamme, A. van den Boogaart, S. Van Huffel, J. Magn. Reson. 129, 35-43 (1997)

35. A. Naressi, C. Couturier, J.M. Devos, M. Janssen, C. Mangeat, R. de Beer, D. Graveron-Demilly, MAGMA 12, 141-152 (2001)

36. M.A. Schroeder, P. Swietach, H.J. Atherton, F.A. Gallagher, P. Lee, G.K. Radda, K. Clarke, D.J. Tyler, Cardiovasc. Res. 86, 82-91 (2010)

37. M.E. Merritt, C. Harrison, A.D. Sherry, C.R. Malloy, S.C. Burgess, Proc. Natl. Acad. Sci. USA. 108, 19084-19089 (2011)

38. K.X. Moreno, S.M. Sabelhaus, M.E. Merritt, A.D. Sherry, C.R. Malloy, Am. J. Physiol. Heart Circul. Physiol. 298, H1556-H1563 (2010)

39. C.R. Malloy, A.D. Sherry, F.M.H. Jeffrey, J. Biol. Chem. 263, 6964-6971 (1988)

40. J.C. Chatham, B. Bouchard, C. Des Rosiers, Mol. Cell. Biochem. 249, 105-112 (2003)

41. R. Southworth, K.A.B. Davey, A. Warley, P.B. Garlick, Am. J. Physiol. Heart Circul. Physiol. 292, H378-H386 (2007)

42. P.E. Larson, A.B. Kerr, A.P. Chen, M.S. Lustig, M.L. Zierhut, S. Hu, C.H. Cunningham, J.M. Pauly, J. Kurhanewicz, D.B. Vigneron, J. Magn. Reson. 194, 121-127 (2008)

43. H. Pedersen, S. Kozerke, S. Ringgaard, K. Nehrke, W.Y. Kim, Magn. Reson. Med. 62, 706-716 (2009) 